TAIWANESE JOURNAL OF MATHEMATICS

Vol. 19, No. 1, pp. 243-252, February 2015

DOI: $10.11650 /$ tjm.19.2015.3873

This paper is available online at http://journal.taiwanmathsoc.org.tw

\title{
MAXIMIZATION AND MINIMIZATION PROBLEMS RELATED TO A $p$-LAPLACIAN EQUATION ON A MULTIPLY CONNECTED DOMAIN
}

\author{
N. Amiri and M. Zivari-Rezapour*
}

\begin{abstract}
In this paper we investigate maximization and minimization problems related to a $p$-Laplacian equation on a multiply connected domain in $\mathbb{R}^{2}$, where the admissible set is a rearrangement class of a fixed function. We prove existence and representation of the maximizers and existence, uniqueness and representation of the minimizer.
\end{abstract}

\section{INTRODUCTION}

Let $\Omega$ be a nonempty, bounded, connected open set in $\mathbb{R}^{2}$ whose boundary is a disjoint union of simple closed curves $\Gamma_{0}, \Gamma_{1}, \ldots, \Gamma_{n}$ of class $C^{2}$, and suppose $\Gamma_{0}$ encloses $\Omega$. Let $1<p<\infty$, we denote the conjugate of $p$ by $p^{\prime}=\frac{p}{p-1}$. We consider the following boundary value problem

$$
\begin{cases}-\Delta_{p} u=f & \text { in } \Omega, \\ u=0 & \text { on } \Gamma_{0}, \\ u=\mathrm{constant} & \text { on } \Gamma_{i}, i=1, \ldots, n, \\ -\int_{\Gamma_{i}}|\nabla u|^{p-2} \nabla u \cdot \mathbf{n} \mathrm{d} s=\gamma_{i} & \text { for } i=1, \ldots, n,\end{cases}
$$

where $f \in L^{p^{\prime}}(\Omega), \mathbf{n}$ is the unit outer normal to $\partial \Omega$, boundary of $\Omega$, and $\gamma_{1}, \ldots, \gamma_{n}$ are real numbers. When $p=2$, G. R. Burton in [4, Appendix] has proved that the problem (1) has exactly one solution. By similar method, we show that (1) still has a unique solution when $1<p<\infty$. For each $f \in L^{p^{\prime}}(\Omega)$ we denote the unique solution of (1) by $u_{f}$. For application of (1), when $p=2$, to fluids dynamics (vorticity) see [4]. Our interest in this paper are in the maximization and minimization of the quantity $\frac{1}{p} \int_{\Omega}\left|\nabla u_{f}\right|^{p} \mathrm{~d} x$, the kinetic energy, as $f$ varies in a rearrangement class of a fixed function in $L^{p^{\prime}}(\Omega)$; see the next section for precise definition of rearrangement of

Received October 6, 2013, accepted June 4, 2014.

Communicated by Franco Giannessi.

2010 Mathematics Subject Classification: 35J20, 49J20.

Key words and phrases: Rearrangement, Maximization, Minimization, Existence, Uniqueness.

*Corresponding author. 
functions. Rearrangement optimization problems have been investigated in recent years by many authors, see $[5,6,7,8,9,10,11,12,13,14,15,16]$. However, the boundary value problem in the one which is discussed here is interesting and very different from others.

\section{REARRANGEMENT}

Let $E$ be a (Lebesgue) measurable set in $\mathbb{R}^{N}$. Real measurable functions $f$ and $g$ on $E$ are rearrangements of each other whenever

$$
\mathcal{L}_{N}(\{x \in \Omega: f(x) \geq \alpha\})=\mathcal{L}_{N}(\{x \in \Omega: g(x) \geq \alpha\}), \text { for all } \alpha \in \mathbb{R},
$$

where $\mathcal{L}_{N}$ denotes the $N$-dimensional Lebesgue measure. It is well known that if $f \in$ $L^{r}(E), 1 \leq r \leq \infty$, and $g$ be a rearrangement of $f$, then $g \in L^{r}(E)$ and in fact $\|f\|_{r}=$ $\|g\|_{r}$, where $\|\cdot\|_{r}$ denotes the standard norm on $L^{r}(E)$. We denote the rearrangement class of $f$ by $\mathcal{R}(f)$ which comprises all functions which are rearrangements of $f$. The readers can see $[2,3]$ for more results about rearrangements of functions.

We now collect some useful lemmas to be applied later.

Lemma 2.1. ([3]). Let $p>1$ and $f_{0} \in L^{p}(E)$. Then

(i) $\overline{\mathcal{R}\left(f_{0}\right)}$, the weak closure of $\mathcal{R}\left(f_{0}\right)$ in $L^{p}(E)$, is compact with respect to $L^{p^{\prime}}$ topology, weak topology, on $L^{p}(E)$.

(ii) $\overline{\mathcal{R}\left(f_{0}\right)}$ is convex.

Lemma 2.2. ([3]). Let $f_{0}: E \rightarrow \mathbb{R}$ and $g: E \rightarrow \mathbb{R}$ be two measurable functions. If every level set of $g$ has measure zero then there exists an increasing function $\xi$ such that $\xi(g) \in \mathcal{R}\left(f_{0}\right)$. Furthermore there exists a decreasing function $\eta$ such that $\eta(g) \in \mathcal{R}\left(f_{0}\right)$.

Lemma 2.3. ([3]). Let $p>1, f_{0} \in L^{p}(E)$ and $g \in L^{p^{\prime}}(E)$.

(i) If there is an increasing function $\xi$ such that $\xi(g) \in \mathcal{R}\left(f_{0}\right)$ then

$$
\int_{E} f g \mathrm{~d} x \leq \int_{E} \xi(g) g \mathrm{~d} x, \quad \text { for all } f \in \overline{\mathcal{R}\left(f_{0}\right)},
$$

and $\xi(g)$ is the unique maximizer relative to $\overline{\mathcal{R}\left(f_{0}\right)}$.

(ii) If there is a decreasing function $\eta$ such that $\eta(g) \in \mathcal{R}\left(f_{0}\right)$ then

$$
\int_{E} f g \mathrm{~d} x \geq \int_{E} \eta(g) g \mathrm{~d} x, \quad \text { for all } f \in \overline{\mathcal{R}\left(f_{0}\right)},
$$

and $\eta(g)$ is the unique minimizer relative to $\overline{\mathcal{R}\left(f_{0}\right)}$. 
Lemma 2.4. ([2]). Let $1 \leq r \leq \infty$ and $s$ be the conjugate exponent of $r$. Let $g \in L^{r}(E)$ and $\Psi: L^{r}(E) \rightarrow \mathbb{R}$ be convex.

(i) Suppose that $\Psi$ is sequentially continuous in the $L^{s}$-topology on $L^{r}(E)$. Then $\Psi$ attains a maximum value relative to $\mathcal{R}(g)$.

(ii) Suppose that $\Psi$ is strictly convex, that $g^{*}$ is a maximizer for $\Psi$ relative to $\mathcal{R}(g)$ and that $w$ is a member of sub-gradient of $\Psi$ at $g^{*}$. Then $g^{*}=\xi(w)$ almost everywhere in $E$ for some increasing function $\xi$.

\section{EXISTENCE AND UNIQUENESS}

In this section we prove the existence and uniqueness for the boundary value problem (1).

Theorem 3.1. Let $\gamma_{1}, \ldots, \gamma_{n} \in \mathbb{R}, 1<p<\infty$, and $f \in L^{p^{\prime}}(\Omega)$. Then the boundary value problem (1) has a unique solution.

Proof. Let $\Omega_{0}, \Omega_{1}, \ldots, \Omega_{n}$ be the regions enclosed by $\Gamma_{0}, \Gamma_{1}, \ldots, \Gamma_{n}$. Let

$$
W=\left\{w \in W^{1, p}(\Omega) \mid w=0 \text { on } \Gamma_{0} \text { and } w=\text { constant on } \Gamma_{i}, i=1, \ldots, n\right\} .
$$

If $w \in W$, then we denote the value of $w$ on $\Gamma_{i}$ by $(w)_{i}$ for $i=1, \ldots, n$. Define

$$
J(w):=\frac{1}{p} \int_{\Omega}|\nabla w|^{p} \mathrm{~d} x-\int_{\Omega} f w \mathrm{~d} x+\sum_{i=1}^{n} \gamma_{i}(w)_{i}, \quad w \in W .
$$

By the trace embedding $W^{1, p}(\Omega) \rightarrow L^{p}(\partial \Omega)$ we infer that $W$ is a closed linear subspace of $W^{1, p}(\Omega)$, and $W$ comprises the restrictions to $\Omega$ of elements of $W_{0}^{1, p}\left(\Omega_{0}\right)$ that are constant on $\Omega_{i}, i=1, \ldots, n$. We consider the equivalent norm for $W_{0}^{1, p}\left(\Omega_{0}\right)$ that is defined as follows

$$
\|w\|=\left(\int_{\Omega_{0}}|\nabla w|^{p} \mathrm{~d} x\right)^{\frac{1}{p}} .
$$

It is well known that the function $x \rightarrow|x|^{p}, x \in \mathbb{R}^{N}$, is strictly convex. From this, it is easy to deduce that $J$ is strictly convex. We know that $J$ is differentiable on $W$ with

$$
J^{\prime}(w) v=\int_{\Omega}|\nabla w|^{p-2} \nabla w \cdot \nabla v \mathrm{~d} x-\int_{\Omega} f v \mathrm{~d} x+\sum_{i=1}^{n} \gamma_{i}(v)_{i} .
$$

Moreover,

$$
J(w) \geq \frac{1}{p}\|w\|^{p}-\|f\|_{p^{\prime}}\|w\|_{p}+\sum_{i=1}^{n} \gamma_{i}(w)_{i} \geq \frac{1}{p}\|w\|^{p}-C\|w\|+\sum_{i=1}^{n} \gamma_{i}(w)_{i},
$$


for some $C>0$. Thus $J$ is coercive because $p>1$. Therefore $J$ has a unique global minimizer. We know that $u \in W$ is a critical point of $J$ whenever $J^{\prime}(u) v=0$, for all $v \in W$. Hence

$$
\int_{\Omega}|\nabla u|^{p-2} \nabla u \cdot \nabla v \mathrm{~d} x-\int_{\Omega} f v \mathrm{~d} x+\sum_{i=1}^{n} \gamma_{i}(v)_{i}=0, \quad \text { for all } v \in W .
$$

Let Lipschitz functions $g^{1}, \ldots, g^{n} \in W$ be chosen to satisfy the boundary conditions $\left(g^{j}\right)_{i}=\delta_{i j}, i, j=1, \ldots, n$. Then (2) is equivalent to

$$
\begin{aligned}
& \int_{\Omega}|\nabla u|^{p-2} \nabla u \cdot \nabla v \mathrm{~d} x-\int_{\Omega} f v \mathrm{~d} x=0, \quad \text { for all } v \in W_{0}^{1, p}(\Omega), \\
& \int_{\Omega}|\nabla u|^{p-2} \nabla u \cdot \nabla g^{j} \mathrm{~d} x-\int_{\Omega} f g^{j} \mathrm{~d} x+\gamma_{j}=0, \quad j=1, \ldots, n .
\end{aligned}
$$

Thus (3) is a variational formulation of $-\Delta_{p} u=f$, in $\Omega$. Now from (4) and Divergence theorem we infer that

$$
\int_{\Omega}\left(-\Delta_{p} u-f\right) g^{j} \mathrm{~d} x+\sum_{i=1}^{n} \int_{\Gamma_{i}} g^{j}|\nabla u|^{p-2} \nabla u \cdot \mathbf{n} \mathrm{d} s+\gamma_{j}=0, j=1, \ldots, n,
$$

which reduces to

$$
\int_{\Gamma_{j}}|\nabla u|^{p-2} \nabla u \cdot \mathbf{n} \mathrm{d} s+\gamma_{j}=0, \quad j=1, \ldots, n .
$$

It follows that (1) holds if and only if $u$ is a critical point of $J$, and therefore (1) has a unique solution.

\section{Optimization Problem}

Let $\gamma_{1}, \ldots, \gamma_{n}$ are fixed real numbers and $1<p<\infty$. Also, let $f_{0}$ is a fixed function in $L^{p^{\prime}}(\Omega)$ and $\mathcal{R}:=\mathcal{R}\left(f_{0}\right)$. It is well known that the solution $u_{f}$ of problem (1) satisfies the following variational problem

$$
\frac{1}{p} \int_{\Omega}\left|\nabla u_{f}\right|^{p} \mathrm{~d} x-\int_{\Omega} f u_{f} \mathrm{~d} x+\sum_{i=1}^{n} \gamma_{i}\left(u_{f}\right)_{i}=\min _{w \in W} J_{f}(w),
$$

where

$$
J_{f}(w):=\frac{1}{p} \int_{\Omega}|\nabla w|^{p} \mathrm{~d} x-\int_{\Omega} f w \mathrm{~d} x+\sum_{i=1}^{n} \gamma_{i}(w)_{i}
$$


By (2), when $v=u=u_{f}$, and (5) we deduce that

$$
\begin{aligned}
(p-1) \int_{\Omega}\left|\nabla u_{f}\right|^{p} \mathrm{~d} x & =\max _{w \in W}\left(-p J_{f}(w)\right) \\
& =\max _{w \in W}\left(p \int_{\Omega} f w \mathrm{~d} x-\int_{\Omega}|\nabla w|^{p} \mathrm{~d} x-p \sum_{i=1}^{n} \gamma_{i}(w)_{i}\right) .
\end{aligned}
$$

We define the functional $\varphi: L^{p^{\prime}}(\Omega) \rightarrow \mathbb{R}$ by

$$
\varphi(f):=\int_{\Omega}\left|\nabla u_{f}\right|^{p} \mathrm{~d} x .
$$

Our interest is in the following optimization problems

$$
\max _{f \in \mathcal{R}} \varphi(f),
$$

and

$$
\min _{f \in \mathcal{R}} \varphi(f) .
$$

We now prove some useful lemmas to be applied later.

Lemma 4.1. The functional $\varphi$ is continuous with respect to weak topology in $L^{p^{\prime}}(\Omega)$.

Proof. Let a sequence $\left\{f_{j}\right\}$ and $f$ be all in $L^{p^{\prime}}(\Omega)$ such that $f_{j} \rightarrow f$ in $L^{p^{\prime}}(\Omega)$. To simplify notation we write $u_{j}$ in place of $u_{f_{j}}$. From (6) we have

(9)

$$
\begin{aligned}
& (p-1) \varphi(f)+p \int_{\Omega}\left(f_{j}-f\right) u_{f} \mathrm{~d} x \\
= & p \int_{\Omega} f_{j} u_{f} \mathrm{~d} x-\int_{\Omega}\left|\nabla u_{f}\right|^{p} \mathrm{~d} x-p \sum_{i=1}^{n} \gamma_{i}\left(u_{f}\right)_{i} \\
\leq & (p-1) \varphi\left(f_{j}\right) \\
= & p \int_{\Omega} f u_{j} \mathrm{~d} x-\int_{\Omega}\left|\nabla u_{j}\right|^{p} \mathrm{~d} x-p \sum_{i=1}^{n} \gamma_{i}\left(u_{j}\right)_{i}+p \int_{\Omega}\left(f_{j}-f\right) u_{j} \mathrm{~d} x \\
\leq & (p-1) \varphi(f)+p \int_{\Omega}\left(f_{j}-f\right) u_{j} \mathrm{~d} x .
\end{aligned}
$$

Since $f_{j} \rightarrow f$ we deduce that

$$
\lim _{j \rightarrow \infty} \int_{\Omega}\left(f_{j}-f\right) u_{f} \mathrm{~d} x=0 .
$$


Now, we prove that

$$
\lim _{j \rightarrow \infty} \int_{\Omega}\left(f_{j}-f\right) u_{j} \mathrm{~d} x=0
$$

From (2), when $v=u=u_{j}, f_{j} \rightarrow f$, Poincarè's inequality for $W_{0}^{1, p}\left(\Omega_{0}\right)$ and Hölder's inequality we find that

$$
\begin{aligned}
\left\|u_{j}\right\|^{p} & \leq\left|\int_{\Omega} f_{j} u_{j} \mathrm{~d} x\right|+\sum_{i=1}^{n}\left|\gamma_{i}\left(u_{j}\right)_{i}\right| \\
& \leq\left\|f_{j}\right\|_{p^{\prime}}\left\|u_{j}\right\|_{p}+C\left\|u_{j}\right\| \\
& \leq C\left\|u_{j}\right\|,
\end{aligned}
$$

where $C$ denotes a positive constant that can change from line to line. Note that in the above inequalities, the second inequality, we used this fact that if $i \in\{1,2, \cdots, n\}$ then

$$
\left|\left(u_{j}\right)_{i}\right|^{p} \mathcal{L}_{2}\left(\Omega_{i}\right)=\int_{\Omega_{i}}\left|u_{j}\right|^{p} \mathrm{~d} x \leq \int_{\Omega_{0}}\left|u_{j}\right|^{p} \mathrm{~d} x \leq C\left\|u_{j}\right\|^{p} .
$$

Hence $\left\{u_{j}\right\} \subset W$ is a bounded sequence in $W_{0}^{1, p}\left(\Omega_{0}\right)$, thus there exists a subsequence, still denoted $\left\{u_{j}\right\}$, that converges weakly to $\hat{u} \in W$, because $W$ is closed. The compact imbedding of $W^{1, p}(\Omega)$ into $L^{p}(\Omega)$ implies that $\left\{u_{j}\right\}$ converges strongly to $\hat{u}$ in $L^{p}(\Omega)$. Thus, we derive (11). Therefore, (9), (10) and (11) complete the proof of the lemma.

Remark 4.1. According to the proof of the Lemma 4.1, we claim that $\hat{u}$ is equal to $u_{f}$ almost every where in $\Omega$. We know that

$$
(p-1) \varphi\left(f_{j}\right)=p \int_{\Omega} f_{j} u_{j} \mathrm{~d} x-\int_{\Omega}\left|\nabla u_{j}\right|^{p} \mathrm{~d} x-p \sum_{i=1}^{n} \gamma_{i}\left(u_{j}\right)_{i} .
$$

Now by the weak lower semicontinuity of the norm $\|\cdot\|$ and (6), we derive

$$
\begin{aligned}
(p-1) \varphi(f) & \leq p \int_{\Omega} f \hat{u} \mathrm{~d} x-\int_{\Omega}|\nabla \hat{u}|^{p} \mathrm{~d} x-p \sum_{i=1}^{n} \gamma_{i}(\hat{u})_{i} \\
& \leq(p-1) \varphi(f) .
\end{aligned}
$$

Therefore, the uniqueness of the maximizer of the functional $-p J_{f}($.$) implies that$ $\hat{u}=u_{f}$ almost every where in $\Omega$.

Lemma 4.2. The functional $\varphi$ is strictly convex in $L^{p^{\prime}}(\Omega)$. 
Proof. Let $0 \leq t \leq 1$ and $f, g \in L^{p^{\prime}}(\Omega)$. For each $w \in W$ we have

$$
\begin{aligned}
-p J_{t f+(1-t) g}(w) & =p \int_{\Omega}(t f+(1-t) g) w \mathrm{~d} x-\int_{\Omega}|\nabla w|^{p} \mathrm{~d} x-p \sum_{i=1}^{n} \gamma_{i}(w)_{i} \\
& =t\left(-p J_{f}(w)\right)+(1-t)\left(-p J_{g}(w)\right) .
\end{aligned}
$$

Hence, from (6) we infer that

$$
\varphi(t f+(1-t) g) \leq t \varphi(f)+(1-t) \varphi(g)
$$

thus $\varphi$ is convex. Now, we show that $\varphi$ is strictly convex. Suppose for some $0<t<1$, we have

$$
\varphi(h)=t \varphi(f)+(1-t) \varphi(g)
$$

where $h:=t f+(1-t) g$. Thus,

$$
J_{h}\left(u_{h}\right)=t J_{f}\left(u_{f}\right)+(1-t) J_{g}\left(u_{g}\right) .
$$

Hence,

$$
t J_{f}\left(u_{h}\right)+(1-t) J_{g}\left(u_{h}\right)=t J_{f}\left(u_{f}\right)+(1-t) J_{g}\left(u_{g}\right) .
$$

Since $0<t<1$, we derive $J_{f}\left(u_{h}\right)=J_{f}\left(u_{f}\right)$ and $J_{g}\left(u_{h}\right)=J_{g}\left(u_{g}\right)$. By the uniqueness of the minimizer of the functionals $J_{f}($.$) and J_{g}($.$) on W$, we deduce that

$$
u_{h}=u_{f}=u_{g}, \text { a.e. in } \Omega \text {. }
$$

Thus, $-\Delta_{p} u_{f}=-\Delta_{p} u_{g}$ almost every where in $\Omega$, so $f=g$ almost every where in $\Omega$. Therefore, $\varphi$ is strictly convex.

Lemma 4.3. Let $f \in L^{p^{\prime}}(\Omega)$. The functional $\varphi$ is Gateaux differentiable at $f$ with derivative

$$
\varphi^{\prime}(f) g=\frac{p}{p-1} \int_{\Omega} g u_{f} \mathrm{~d} x
$$

for all $g \in L^{p^{\prime}}(\Omega)$.

Proof. Let $\left\{t_{j}\right\}$ be a sequence of positive numbers that tends to zero. Let $f, g \in L^{p^{\prime}}(\Omega)$ and $h_{j}:=f+t_{j}(g-f), j \geq 1$. So, $h_{j} \rightarrow f$ in $L^{p^{\prime}}(\Omega)$ as $j \rightarrow 0$. From (9) we have

$$
\begin{aligned}
(p-1) \varphi(f)+p \int_{\Omega}\left(h_{j}-f\right) u_{f} \mathrm{~d} x & \leq(p-1) \varphi\left(h_{j}\right) \\
& \leq(p-1) \varphi(f)+p \int_{\Omega}\left(h_{j}-f\right) u_{j} \mathrm{~d} x,
\end{aligned}
$$


where $u_{j}:=u_{h_{j}}$. Thus,

$$
\frac{p}{p-1} \int_{\Omega}(g-f) u_{f} \mathrm{~d} x \leq \frac{\varphi\left(f+t_{j}(g-f)\right)-\varphi(f)}{t_{j}} \leq \frac{p}{p-1} \int_{\Omega}(g-f) u_{j} \mathrm{~d} x
$$

As a consequence of Remark 4.1, $u_{j} \rightarrow u_{f}$ in $L^{p}(\Omega)$. This coupled with (12), implies that

$$
\lim _{j \rightarrow \infty} \frac{\varphi\left(f+t_{j}(g-f)\right)-\varphi(f)}{t_{j}}=\frac{p}{p-1} \int_{\Omega}(g-f) u_{f} \mathrm{~d} x .
$$

Therefore, the proof of the lemma follows.

Now, we are ready to prove the main results of this section.

Theorem 4.4. The maximization problem (7) is solvable; that is, there exists $f^{*} \in$ $\mathcal{R}$ such that

$$
\varphi\left(f^{*}\right)=\max _{f \in \mathcal{R}} \varphi(f) .
$$

Moreover, there exists an increasing function $\xi$ such that $f^{*}=\xi\left(u_{f *}\right)$ almost everywhere in $\Omega$.

Proof. From Lemma 4.1, Lemma 4.2 and Lemma 2.4(i) we infer that there exists $f^{*} \in \mathcal{R}$ such that $\varphi(f) \leq \varphi\left(f^{*}\right)$, for all $f \in \mathcal{R}$. From Lemma 4.3, $\varphi(f)$ is Gâteaux differentiable with derivative $\frac{p}{p-1} u_{f}$. Since $\varphi$ is strictly convex, by Lemma 2.4(ii), there is an increasing function $\xi$ such that $f^{*}=\xi\left(u_{f^{*}}\right)$.

Theorem 4.5. If $f_{0}>0$ in $\Omega$, then the minimization problem (8) has a unique solution. Moreover, if $f_{*}$ be the minimizer, then $f_{*}=\eta\left(u_{f_{*}}\right)$ for some decreasing function $\eta$.

Proof. We know $\varphi$ is weakly continuous in $L^{p^{\prime}}(\Omega)$, Lemma 4.1, and $\overline{\mathcal{R}}$ is weakly compact, Lemma 2.1. Thus, there exists $f_{*} \in \overline{\mathcal{R}}$ such that

$$
\varphi\left(f_{*}\right)=\min _{f \in \overline{\mathcal{R}}} \varphi(f) .
$$

Since $\varphi$ is strictly convex, Lemma 4.2 , we infer that $f_{*}$ is unique. Now, we prove that $f_{*} \in \mathcal{R}$. From Lemma 2.14 of [3] we have

$$
\mathcal{L}_{2}\left(\left\{x \in \Omega: f_{*}(x)>0\right\} \geq \mathcal{L}_{2}\left(\left\{x \in \Omega: f_{0}(x)>0\right\}=\mathcal{L}_{2}(\Omega),\right.\right.
$$

so, $f_{*}>0$ in $\Omega$. This coupled with $-\Delta_{p} u_{f_{*}}=f_{*}$ in $\Omega$, implies that every level set of $u_{f_{*}}$ in $\Omega$ has measure zero. By applying Lemma 2.2 we derive that there exists a decreasing function $\eta$ such that $\eta\left(u_{f_{*}}\right) \in \mathcal{R}$. Now, from Lemma 2.3(ii) we have

$$
\int_{\Omega} f u_{f_{*}} \mathrm{~d} x \geq \int_{\Omega} \eta\left(u_{f_{*}}\right) u_{f_{*}} \mathrm{~d} x, \text { for all } f \in \overline{\mathcal{R}} .
$$


Let $0<t<1$ and $f \in \overline{\mathcal{R}}$. We define $f_{t}:=t f+(1-t) f_{*}$. Since $\overline{\mathcal{R}}$ is convex, Lemma 2.1(i), $f_{t} \in \overline{\mathcal{R}}$ for all $0<t<1$. From Lemma 4.3, for sufficiently small $t$ we have

$$
\varphi\left(f_{*}\right) \leq \varphi\left(f_{t}\right)=\varphi\left(f_{*}\right)+\frac{t p}{p-1} \int_{\Omega}\left(f-f_{*}\right) u_{f_{*}} \mathrm{~d} x+o(t) .
$$

Thus, when $t \rightarrow 0^{+}$we deduce

$$
\int_{\Omega} f u_{f_{*}} \mathrm{~d} x \geq \int_{\Omega} f_{*} u_{f_{*}} \mathrm{~d} x, \text { for all } f \in \overline{\mathcal{R}}
$$

Therefore, by (13), (14) and Lemma 2.3(ii) we derive $f_{*}=\eta\left(u_{f_{*}}\right)$.

\section{REFERENCES}

1. R. Adams, Sobolev Spaces, Academic Press, New York, 1975.

2. G. R. Burton, Rearrangements of functions, maximization of convex functionals, and vortex rings, Math. Ann., 276 (1987), 225-253.

3. G. R. Burton, Variational problems on classes of rearrangements and multiple configurations for steady vortices, Ann. Inst. Henri Poincare, 6 (1989), 295-319.

4. G. R. Burton, Rearrangements of functions, saddle points and uncountable families of steady configurations for a vortex, Acta Math., 163 (1989), 291-309.

5. F. Cuccu, B. Emamizadeh and G. Porru, Optimization of the first eigenvalue in problems involving the $p$-Laplacian, Proc. Amer. Math. Soc., 137(5) (2009), 1677-1687.

6. F. Cuccu, B. Emamizadeh and G. Porru, Optimization problems for an elastic plate, $J$. Math. Phys., 47(8) (2006), 082901, 12 pp.

7. F. Cuccu, B. Emamizadeh and G. Porru, Nonlinear elastic membranes involving the p-Laplacian operator, Electron. J. Differential Equations, 49 (2006), 10 pp.

8. F. Cuccu, G. Porru and S. Sakaguchi, Optimization problems on general classes of rearrangements, Nonlinear Analysis, 74 (2011), 5554-5565.

9. F. Cuccu, G. Porru and A. Vitolo, Optimization of the energy integral in two classes of rearrangements, Nonlinear Stud., 17(1) (2010), 23-35.

10. L. M. Del Pezzo and J. Fernández Bonder, Some optimization problems for $p$-Laplacian type equations, Appl. Math. Optim., 59(3) (2009), 365-381.

11. L. M. Del Pezzo and J. Fernández Bonder, Remarks on an optimization problem for the p-Laplacian, Appl. Math. Lett., 23(2) (2010), 188-192.

12. B. Emamizadeh and M. Zivari-Rezapour, Optimization of the principal eigenvalue of the pseudo $p$-Laplacian operator with Robin boundary conditions, International Journal of Mathematics, 23(12) (2012), 1250127, 17 pp. 
13. B. Emamizadeh and M. Zivari-Rezapour, Rearrangements and minimization of the principal eigenvalue of a nonlinear Steklov problem, Nonlinear Anal., 74(16) (2011), 56975704.

14. B. Emamizadeh and M. Zivari-Rezapour, Rearrangement optimization for some elliptic equations, J. Optim. Theory Appl., 135(3) (2007), 367-379.

15. B. Emamizadeh and J. V. Prajapat, Maximax and minimax rearrangement optimization problems, Optim. Lett., 5(4) (2011), 647-664.

16. M. Zivari-Rezapour, Maximax rearrangement optimization related to a homogeneous Dirichlet problem, Arab. J. Math., 2(4) (2013), 427-433, DOI 10.1007/s40065-0130083-0.

N. Amiri and M. Zivari-Rezapour

Department of Mathematics

Faculty of Mathematical Sciences \& Computer

Shahid Chamran University

Golestan Blvd. Ahvaz

Iran

E-mail: n-amiri@phdstu.scu.ac.ir

mzivari@scu.ac.ir 